\title{
The impact of patient advisors on healthcare outcomes: a systematic review
}

Anjana E. Sharma ${ }^{1 *}$, Margae Knox $^{1}$, Victor L. Mleczko ${ }^{2}$ and J. Nwando Olayiwola ${ }^{3}$

\begin{abstract}
Background: Patient advisory councils are a way for healthcare organizations to promote patient engagement. Despite mandates to implement patient advisory councils through programs like the Patient-Centered Medical Home (PCMH), there is a paucity of data measuring the impact of patients functioning in advisory roles. Our objective is to investigate whether patient engagement in patient advisory councils is linked to improvements in clinical quality, patient safety or patient satisfaction.

Methods: We searched PubMed, SCOPUS, CINAHL and Google Scholar for English language publications between November 2002 to August 2015, using a combination of "patient advisor" and "care outcomes" search terms. Article selection utilized dual screening facilitated by DistillerSR software, with group discussion to resolve discordance. Observational studies, randomized controlled trials, and case studies were included that described patients serving in an advisory role where primary outcomes were mentioned. Reference lists of included studies and grey literature searches were conducted. Qualitative thematic analysis was performed to synthesize results.
\end{abstract}

Results: Database searching yielded 639 articles total after removing duplicates, with 129 articles meeting full text inclusion criteria. 32 articles were identified for final review, 16 of which were case studies. Advisory roles included patient advisory councils, ad-hoc patient committees, community advisory councils, experience-based co-design, and other. Four practice-based studies from one research group, involving community advisors in the design of public health interventions, found improved clinical outcomes. No prospective experimental studies assessed the impact of patient advisors on patient safety or patient satisfaction. One cluster-randomized RCT showed that patient advisors helped health care planning efforts identify priorities more aligned with the PCMH. Ten case studies reported anecdotal benefit to individual patient advisors.

Conclusion: Five included studies demonstrate promising methods for evaluating patient engagement in healthcare delivery and describe impacts on clinical outcomes and priority setting. Based on the case studies found, patient advisors tend to contribute to patient-facing services that may affect clinical care but are not easily evaluated. As clinics and hospitals implement patient advisory councils, rigorous evaluation of their programs is needed to support the expansion of system-level patient engagement.

Trial registration: This systematic review was registered in the PROSPERO database of the University of York Centre for Reviews and Dissemination (ID: 2015:CRD42015030020).

Keywords: Patient engagement, Patient advisory councils, Experience of care, Practice improvement

\footnotetext{
*Correspondence: Anjana.sharma@ucsf.edu

${ }^{1}$ Center for Excellence in Primary Care, Department of Family \& Community

Medicine, UCSF, 995 Potrero Ave, Ward 83, San Francisco, CA 94110, USA

Full list of author information is available at the end of the article
}

(c) The Author(s). 2017 Open Access This article is distributed under the terms of the Creative Commons Attribution 4.0 International License (http://creativecommons.org/licenses/by/4.0/), which permits unrestricted use, distribution, and reproduction in any medium, provided you give appropriate credit to the original author(s) and the source, provide a link to the Creative Commons license, and indicate if changes were made. The Creative Commons Public Domain Dedication waiver (http://creativecommons.org/publicdomain/zero/1.0/) applies to the data made available in this article, unless otherwise stated. 


\section{Background}

Patient engagement can be defined as active partnership between patients, families and caregivers working together to improve healthcare delivery [1]. Patient engagement can be fostered at the individual level, clinic or organizational level, and the policy level [2]. While promoting patient engagement in individual care through self-management is better understood, less is known about clinic or organizational level patient engagement [3]. Examples of clinic or organizational-level patient engagement include patients serving on patient advisory councils, becoming members of quality improvement committees, or participating in training staff [4]. These activities allow healthcare leadership to incorporate the patient perspective and care experience when considering new clinic initiatives, quality improvement projects, or community needs.

Working with patient advisors is a promising method for achieving ongoing system-level patient engagement that can integrate with clinic quality improvement initiatives and address patient experience of care. Patient advisors are patients who meet on a regular basis with clinic staff to improve care delivery at the clinic, hospital, or organizational level. In the United States, patient advisory councils are increasingly mandated within organizations to demonstrate a commitment to patient-centered care, such as the Patient-Centered Medical Home (PCMH) delivery model, which delivers team-based, data-driven and coordinated care [5]. Patient advisory councils are an optional criteria of the 2014 US National Committee for Quality Assurance (NCQA) Patient-Centered Medical Home recognition standards, which is a national accreditation program that awards PCMH certification [6]. Patient and family advisors are also a requirement within US Accountable Care Organizations, which are groups of doctors, hospitals, and other providers who coordinate spending in order to provide quality-based care for a designated group of patients rather than service-based care, in order to share in cost savings for reduced unnecessary care [7]. In the United Kingdom, patient involvement has a historical precedent of patient participation groups providing input for NHS primary care services since the 1970's [8]. In Australia, trained consumer representatives sit on most medical committees [9] and consumer consultant positions have been developed in mental health services [10].

A strong ethical rationale, based on principles of equity and transparency, is often cited in efforts to improve patient engagement through programs such as patient advisory councils or input from patient representatives [11]. However, there has been a lag in the evidence base assessing for any objective benefits of patient engagement at the clinic or organizational level. A systematic review conducted in 2002 by Crawford et al. identified 42 papers published between
1966 and 2000, 31 of which were case studies [12]. Their analysis reported anecdotal findings that patient input at the clinic level seemed to improve readability of educational materials and improve staff attitudes towards patients; these studies lacked formal prospective or pre-post evaluation of patient advisory activities. Another systematic review examined the impact of public involvement in articles published from 1997 to 2009, but only focused on sites in the UK [13]. Our systematic review seeks to update Crawford et al.'s 2002 review to provide a current understanding of the impact of patient advisory councils on concrete healthcare outcomes with an international scope. Initiating and maintaining a patient advisory council or other forms of patient engagement requires a commitment of staffing and other resources, thus clinics and hospital leaders may be resistant to investing in patient engagement without clear evidence to support their benefit. Our primary aim is to investigate the impact of interventions involving patient advisory councils on clinical care outcomes, patient safety, and patient satisfaction, compared to care that doesn't involve patient advisors, for participants at all healthcare settings. Our secondary aim is to survey the impact patient advisors have on healthcare changes such as prioritysetting, patient materials, and impacts on patient advisors themselves.

\section{Methods}

We conducted database search queries targeting articles involving patients serving in an advisory capacity in which authors mentioned clinical care, patient safety or patient satisfaction outcomes. This systematic review followed PRISMA guidelines (PRISMA checklist included in Additional file 1) and was registered in the PROSPERO database of the University of York Centre for Reviews and Dissemination (ID: 2015:CRD42015030020). The initial literature search was conducted from September-October 2015. Reference lists were further searched for relevant articles from October 2015 through May 2016.

\section{Identification of data sources}

We searched PubMed, SCOPUS, CINAHL and Google Scholar databases. The search strategy included a combination of either patient engagement or patient advisory council terms as well as quality outcome terms [Table 1]; the initial PubMed search strategy was then adapted for SCOPUS, CINAHL and Google Scholar, with syntax and search specifications optimized for each search engine (see Additional file 2). Given that much of the work around patient engagement is shared in white papers or non-peer-reviewed publications, we also conducted grey literature searches in order to identify informally published or ongoing patient engagement research. Specific sources of grey literature included white papers available from the Institute for Patient and Family-Centered Care 
Table 1 PubMed Search Strategy

(((Patient OR patients OR consumer OR consumers OR community OR communities) AND ("patient participation"[mh] OR "consumer participation"[mh] OR "patient engagement" OR "consumer engagement" OR "patient participation" OR "consumer participation" OR "consumer involvement" OR "patient involvement"))

$\mathrm{OR}$

("Advisory committees"[mh] OR "Governing board"[mh])) OR ("patient advisory council" OR "patient advisory committee" OR "patient and family advisory council" OR "consumer advisory council" OR "patient advisory committee" OR "consumer advisory boards" OR "Community advisory board" OR "Community advisory council" OR "Community advisory committee" OR "Community advisory boards" OR

"Community advisory council")

AND

("Patient Harm"[Mesh] OR "Patient Safety"[Mesh] OR "Quality of Health Care"[Mesh] OR "Patient Satisfaction"[mh] OR "patient safety" OR "quality of care" OR "clinical outcomes" OR "patient experience" OR "patient satisfaction" OR "consumer satisfaction" OR "Community health planning" OR "population health" OR "Health Care Costs"[Mesh] OR "cost of care" OR "health care costs" OR "Health Priorities/organization and administration" $[\mathrm{mh}]$ OR "health care priority" $[$ Tiab] OR "health care priorities"[Tiab] OR "healthcare priority"[Tiab] OR "healthcare priorities" [Tiab] OR "health priority"[Tiab] OR "health priorities"[Tiab] "Quality Improvement/organization and administration"[mh])

and the Patient-Centered Primary Care Collaborative. Reference lists of our final list of included studies were reviewed and abstracts of relevant articles were reviewed in a similar process. Identified studies were uploaded into DistillerSR, a web-based systematic review software [14].

\section{Study selection}

Two reviewers from the study team, one lead (AES) and one support researcher (VM or MK), independently reviewed titles and abstracts using DistillerSR. Included studies had to describe a patient advisory council activity or intervention, defined for the purpose of this study as a group of patients or consumers working with healthcare staff in order to provide input on healthcare services or delivery. Included studies also had to describe an impact on our primary or secondary outcomes of interest. Our primary outcomes of interest were clinical quality of care, patient safety, or patient experience of care. Our secondary outcomes of interest included other impacts of patient engagement activities to clinic services, policies, priorities, clinical physical space, or impacts on clinic staff or patient advisors themselves. We included randomized controlled trials, observational studies including cross-sectional surveys and qualitative assessments, and case studies. As the intention was to update the Crawford systematic review on patient engagement outcomes, the search query was limited to English language articles dated from November 2002 to August 2015. Inclusion criteria spanned all healthcare settings, including primary care, ambulatory specialty care, inpatient care, emergency department and long-term care. We excluded reports detailing patient engagement within their own individual care, patient engagement within research studies, as well as perspective, policy and protocol studies. A complete list of inclusion criteria is available in Table 2 .

Inclusion-exclusion conflicts were managed by direct discussion and review with an independent fourth senior researcher (NO). If there was a discrepancy, the full text was reviewed and discussed as a group until consensus was reached. Included abstracts were then reviewed in full-text data abstraction. Weighted overall kappa for abstract screen was 0.63 and for full text abstraction was 0.68 , levels which indicates good agreement.

Table 2 Inclusion and Exclusion Criteria

\begin{tabular}{ll}
\hline Inclusion Criteria & Exclusion Criteria \\
\hline Published between November 2002-August 2015 & Published before November 2002 or after August 2015 \\
English language & Non-English publication \\
Healthcare-focused; including but not limited to inpatient and & Study not located in a healthcare setting \\
outpatient, primary care, ambulatory sub-specialty, and &
\end{tabular}
emergency department

Involves patient input on an advisory council, board, or committee

Reports measure of patient engagement impact either for primary outcomes: clinical outcome measure or an NQMC safety or patient satisfaction measure for staff or patients; or secondary outcomes: other impacts on clinic processes, priorities, physical space or impacts on clinic staff or patient advisors themselves

Patient engagement activities described address organization/ system-level changes

Is a research study or case report that includes assessment of patient engagement impact
Did not involve patient input via a council, board or committee

Description of a patient engagement intervention that does not address primary or secondary outcomes.

Exclusively addresses engagement in individual care such as shared decision making or patient activation processes; or involved patient engagement within a research protocol without patient engagement pertaining to the intervention itself

Is a perspective, policy piece, or protocol. 


\section{Data extraction}

Primary outcomes included clinical care quality measures as defined by the National Quality Measures Clearinghouse [15], patient safety measures and patient satisfaction measures reported by either clinical staff or patients and families. Secondary outcomes were other descriptions of patient advisory council impact on the healthcare setting. Both quantitative and qualitative markers of patient engagement effects were extracted using dual-review and an extraction form built in DistillerSR. We developed a data extraction form that collated study type, demographics of each study site (e.g., location, patient population, type of clinical practice), type of patient engagement intervention, outcome metric(s), a summary of the impact patient engagement had on that measure and possible study confounders, and limitations or conflicts of interest.

\section{Analysis}

We applied a thematic analysis approach to synthesize review findings by type of patient advisory council and impacts based on prior systematic reviews that applied a similar approach to heterogeneous study results, rather than attempt a meta-analysis of aggregate data. Study quality was initially determined using the Cochrane Collaborative Bias Assessment Tool [16]. In order to assess quality for both qualitative and quantitative studies, we subsequently used the Mixed Methods Appraisal Tool, which rates studies on a scale from 0 to 4 (Additional file 3) [17].

\section{Results}

Database searching yielded 639 articles after duplicate removal. After abstract review, 129 articles were assessed for full-text data screening and abstraction. After full-text review, 93 articles were excluded yielding 36 articles. Of the 36 articles, one study was eliminated because it did not report our primary or secondary outcomes [18]. One study was eliminated because it was a brief report of a patient advisory council already described in detail in another included study [19]. Two studies were eliminated as they described community participation in regional public health governance in low-resource settings, without clearly referencing if patients were serving as advisors within a healthcare facility setting $[20,21]$. We ultimately included 32 papers for final qualitative synthesis. A full PRISMA diagram of included studies is provided in Fig. 1.

Of included studies, 15 were based in the United Kingdom, 8 in the United States, 4 in Canada, 4 in Australia or New Zealand, and 1 in Sweden (see Table 3). One study was a cluster-randomized controlled trial, 4 described a set of quasi-experimental quality improvement initiatives from one practice group, 2 were systematic reviews, 1 was a cross-sectional survey, and 9 were qualitative or ethnographic studies. The majority $(N=16)$ were case studies comprising a description of a patient advisory project without formal quantitative, qualitative or ethnographic data included. The primary mode of patient advisor intervention was a patient advisory council, referring to a group of patients who met with staff on a regular basis to discuss healthcare improvement activities. Patient engagement was also described in studies as community advisory councils (4 from one institution), experiencebased co-design projects, ad-hoc patient committees who met for a single project, and "other" activities such as "mental health user groups" composed of patients who advise healthcare trusts in the UK. Only one study qualified as having high quality and low risk of bias per the Cochrane Assessment tool [22]; the majority of studies were classified as "not applicable" for quality assessment due to their case-based nature or lack of prospective study design; eight of the studies scored the maximum 4/4 using the Mixed Methods Appraisal Tool. Full summaries of studies including design, intervention, findings and quality rating are included in Table 4.

\section{Primary outcomes: Clinical care, patient safety, and patient satisfaction \\ Clinical care}

No studies reported results from a prospective, randomized controlled trial with respect to our primary outcome of patient advisors impacting clinical care, patient safety, or patient satisfaction. We found four papers describing quasi-experimental public health interventions from one group in Colorado (United States) in which a community advisory council participated in a regional quality improvement campaign to publicize colon cancer screening, asthma, and blood pressure control within a practice-based research network. The community advisory council worked in an iterative process to "translate" health care promotions into public service messages that would be understandable to the lay community [23]. In accompanying articles, this approach was found to have positive results in several distinct interventions and was associated with statistically significant increased selfreported intention to engage in colorectal cancer screening [24], increased use of asthma inhalers as well as asthma action plans [25], and improved blood pressure control rates [26].

Six papers reported case-based or anecdotal findings that patient advisory councils had a role in improving appointment access for patients, which is a domain of care quality. In one case study, breast cancer patients in an experience-based co-design program recommended a change in the scheduling process for newly diagnosed patients [27]. Another case study described how patient and family advisory councils improved their hospital's appointment scheduling so that the scheduling center 


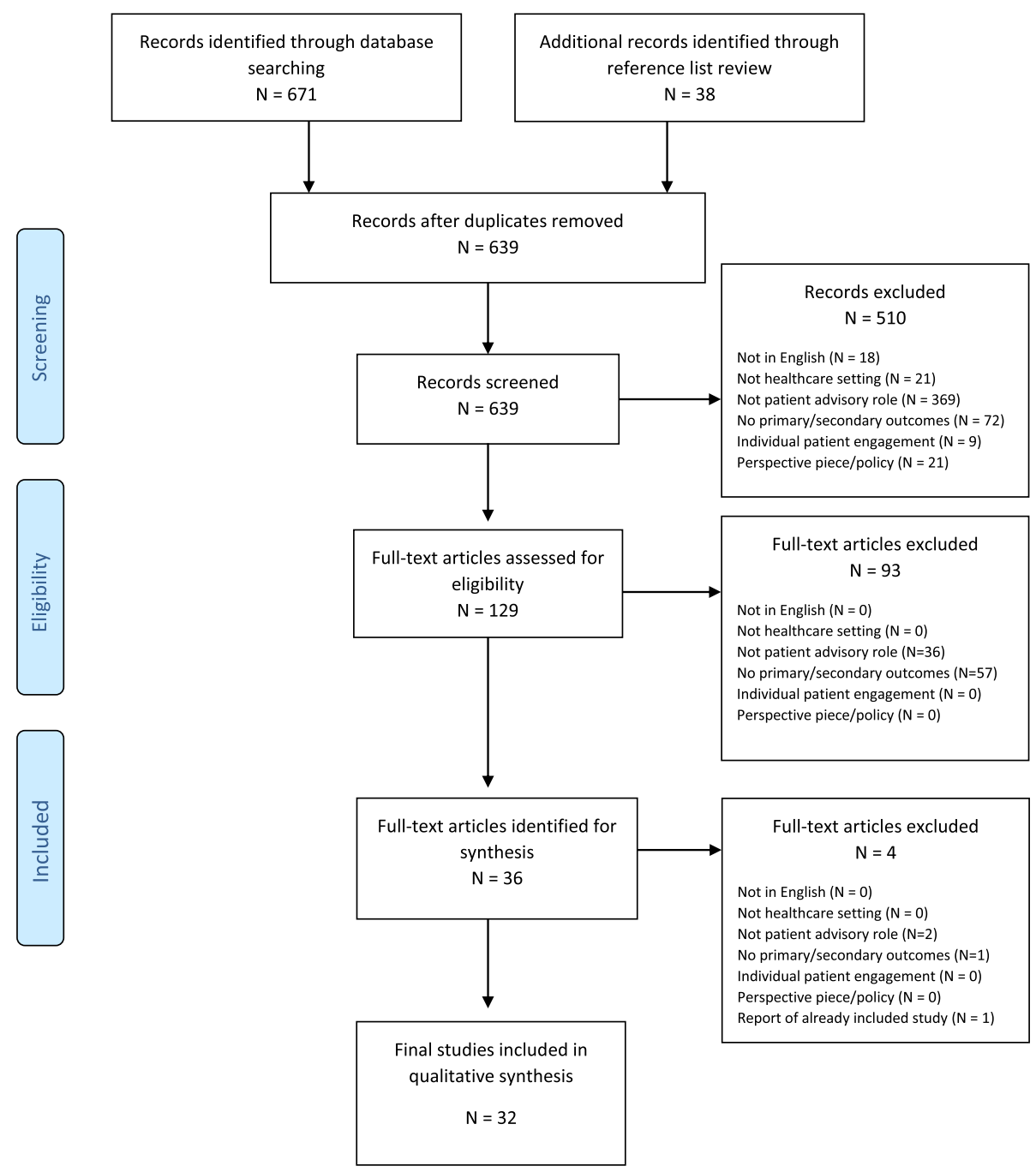

Fig. 1 Prisma 2009 Flow Diagram

contacted patients directly [28]. This change reportedly reduced the number of rescheduled appointments without a summary estimate provided. In the four remaining articles, patient advisory councils were involved in developing a "fast-track" system for an emergency department, improving public transportation to a clinic, and creating drop-in and evening clinic hours. [29-32].

\section{Patient safety}

One report included several case studies describing how patient advisory councils have had a role in patient safety. Examples included a United States hospital in Seattle, Washington that embedded patient advisory committees throughout the institution and attributed this participation to a reduction in falls and medical errors, however specific data on falls and medical errors were not reported. The report described another hospital initiative involving patient advisors in the redesign of their hospital, and attributed this redesign to a reduction in medical errors by $62 \%$ [33].

\section{Patient satisfaction}

Four papers described case-based results that patient advisory councils had a role in affecting patient satisfaction. One hospital's mean patient satisfaction increased from the 10th to the 99th percentile; another hospital's patient satisfaction scores climbed from 95\% to 98\%; these were both attributed to an investment in "patientcentered care" which included patient advisory councils as well as other programs [33]. In another article, a hospital system implemented patient advisory councils as part of a multi-pronged "Interprofessional Collaborative Practice Model" and performed pre-post analysis of patient satisfaction data in four units. The case study reported a positive trend in patient agreement that "I feel I have been listened to by the healthcare team," although 
Table 3 Categories of Included Articles

\begin{tabular}{ll}
\hline Type of Patient Involvement & $\mathrm{N}$ \\
\hline Patient Advisory Council & 11 \\
Community Advisory Council & 4 \\
Ad-hoc Patient Committee & 8 \\
Experience-based co-design & 4 \\
Other & 5 \\
Article Type & \\
Randomized Controlled Trial & 1 \\
Quasi-Experimental Study & 4 \\
Systematic Review & 1 \\
Cross-Sectional Survey & 1 \\
Qualitative Study & 9 \\
Case Study & 16 \\
Location & \\
UK & 15 \\
US & 8 \\
Australia/NZ & 4 \\
Canada & 4 \\
Other (Sweden) & 1 \\
All Articles & 32 \\
\hline
\end{tabular}

statistical analysis was not provided [34]. A patient and family advisory council in a pediatric oncology hospital in Boston MA implemented an ED "fast track" to expedite hematology-oncology admissions, which they attributed to an improved but unreported rate of patient satisfaction for oncology patients and their families [29]. A news article from a hospital in the UK shared how a hospital system trust invited patients who had submitted complaints to participate in regular meetings over two years to brainstorm improvements. Since implementing this program, complaints decreased from 117 to 48 in one year [35].

\section{Secondary outcomes: Clinic priorities, educational materials, physical improvements \\ Patients \& health care priority setting}

The most rigorous study found in this review was a cluster-randomized RCT based in Canada that compared an intervention arm, in which a random selection of regional primary care staff leaders worked with patients to identify priorities, against a control arm in which staff selected primary care priorities without patients. Priorities chosen by staff and patients working together were more aligned with the components of the Patient Centered Medical Home and the Chronic Care Model $(p<0.01)$, although the study could not assess whether these recommendations would be implemented by local primary care officials [22]. Six studies had case-based descriptions of how patient advisors helped to identify hospital or clinic priorities [12, 30, 31, 36, 37].

\section{Benefits for patients and staff}

Ten studies described case-based evidence that participation in an advisory council had a therapeutic or positive benefit for the patients themselves $[8,9,36,38-44]$. A report of interviews with members of a children's hospital teen advisory council found that participation fostered organizational skills and professional development [40]. A qualitative impact assessment of a panel of service users for a Montreal, Canada psychiatric institute interviewed the users serving in an advisory capacity. Users described improved mental health, enhanced education about services, and reduction of perceived stigma due to their involvement [36]. Five studies described how patient advisory councils increased hospital or clinic staff awareness of the patient perspective and patient-centered care $[13,36,40,43,44]$.

\section{Other impacts}

Seventeen studies described how patient advisors helped develop materials for patient education or selfmanagement [8, 9, 24-28, 30, 31, 40, 42, 45-50]. Fifteen studies described how patient advisors recommended changes to a healthcare setting's physical space, such as an improved waiting room, improved accommodations for physical disabilities, or improved layout [13, 27-30, $32,33,35,40,41,48-52]$. Other studies described patient advisor involvement in workflow or service changes $[27,29,30,32,34,44,52]$, patient-developed trainings for staff or trainees [27-31, 33, 42, 44, 49, 51], conference attendance, and web portal improvement [33].

One included study was another systematic review on the impact on patient involvement, but focused on care in the United Kingdom; the studies included in this review comprised narrative, case based evidence describing patient advisors contributing to our secondary outcomes such as improving physical space, expanding clinical services, devising educational materials, and changing healthcare staff attitudes or culture towards the patient perspective [13].

\section{Discussion}

To our knowledge, this is the first systematic review with a focus on patient advisors, with an emphasis on quantifiable care quality outcomes. We did not find any rigorous, prospective RCTs that assessed our primary outcomes of patient clinical care, patient safety, or patient satisfaction. We did identify a group of quasi-experimental studies from one large regional initiative in which community advisory councils aided in the development of patientcentered messaging about the importance of colorectal 


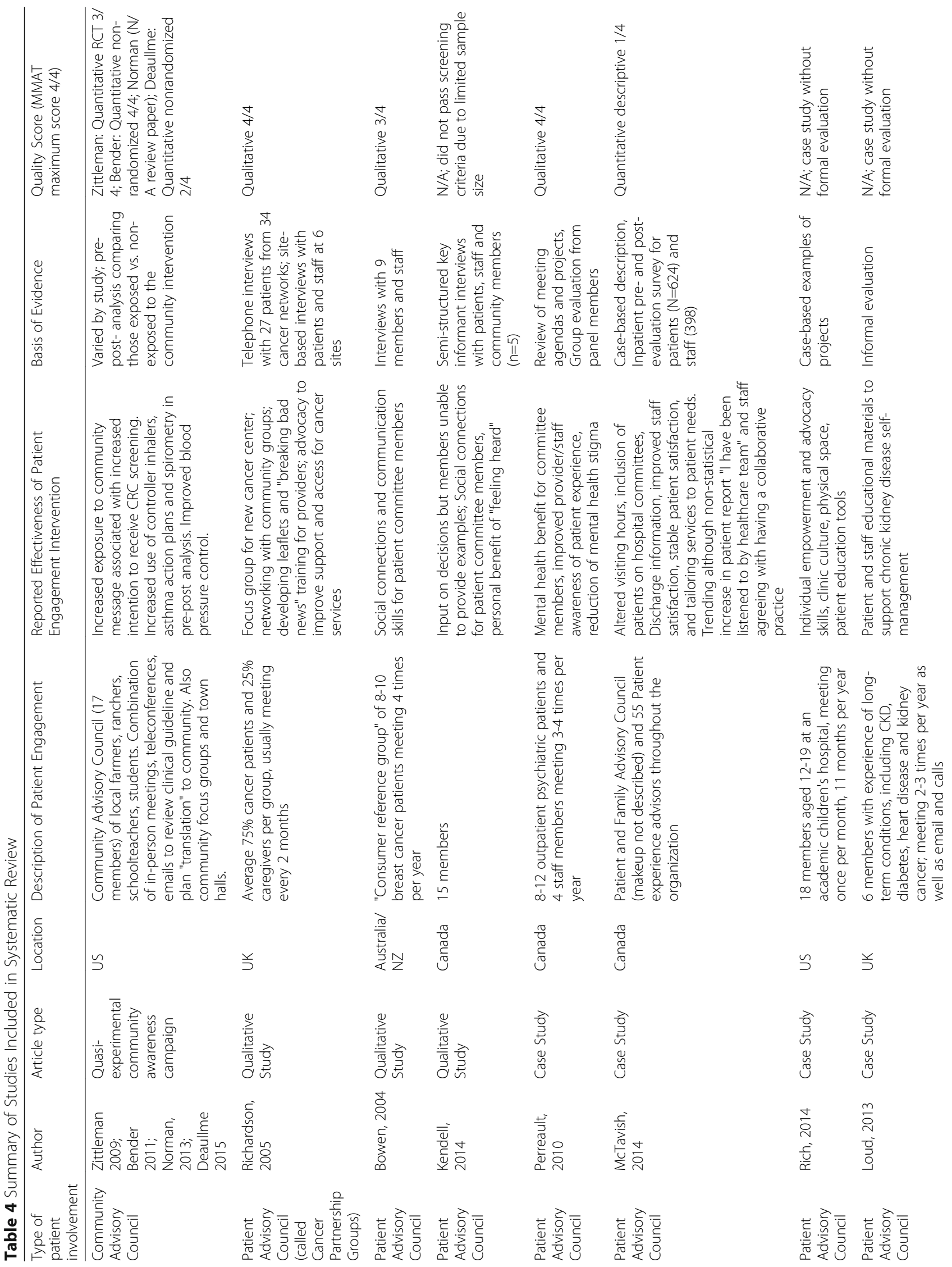




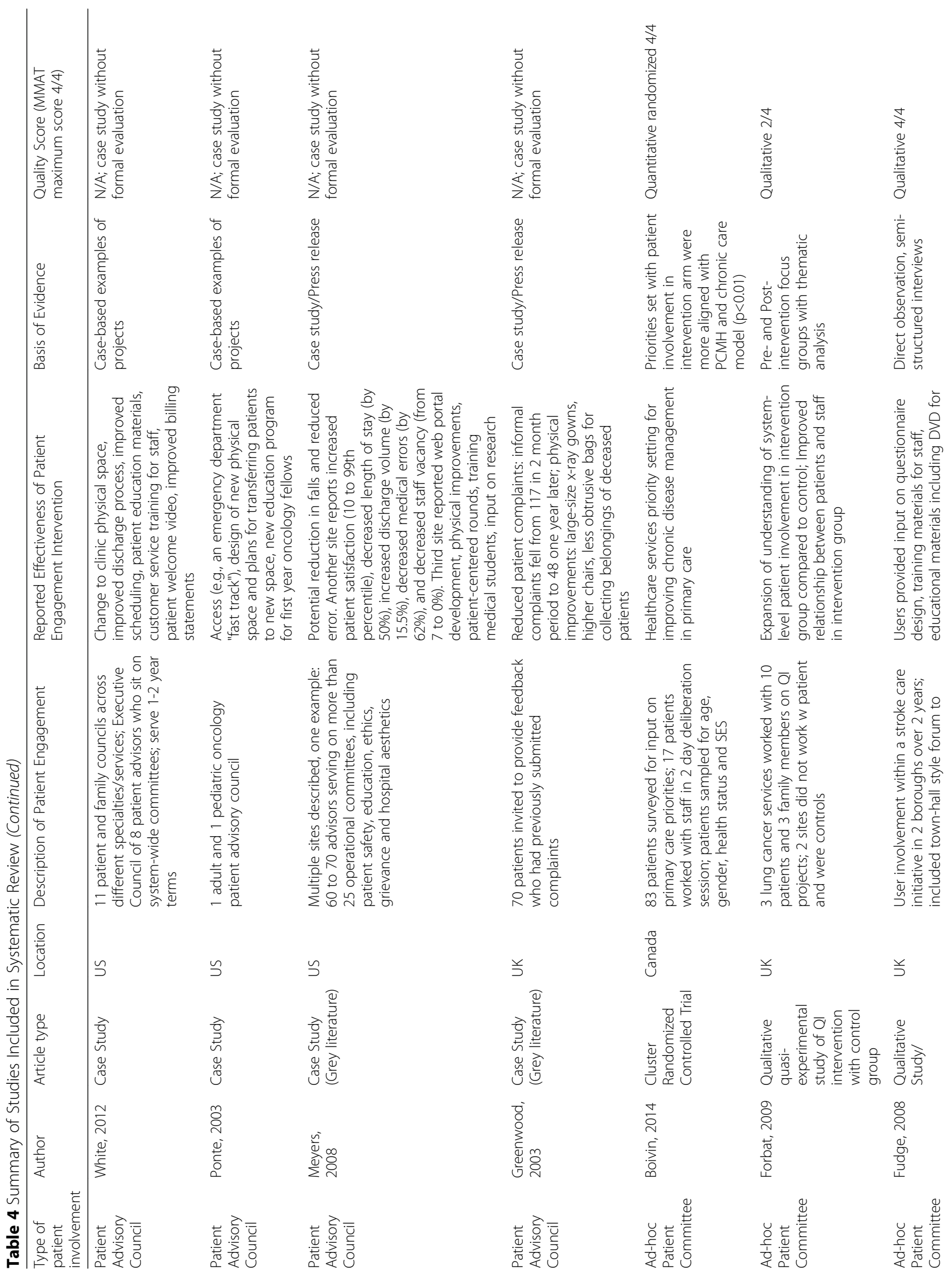




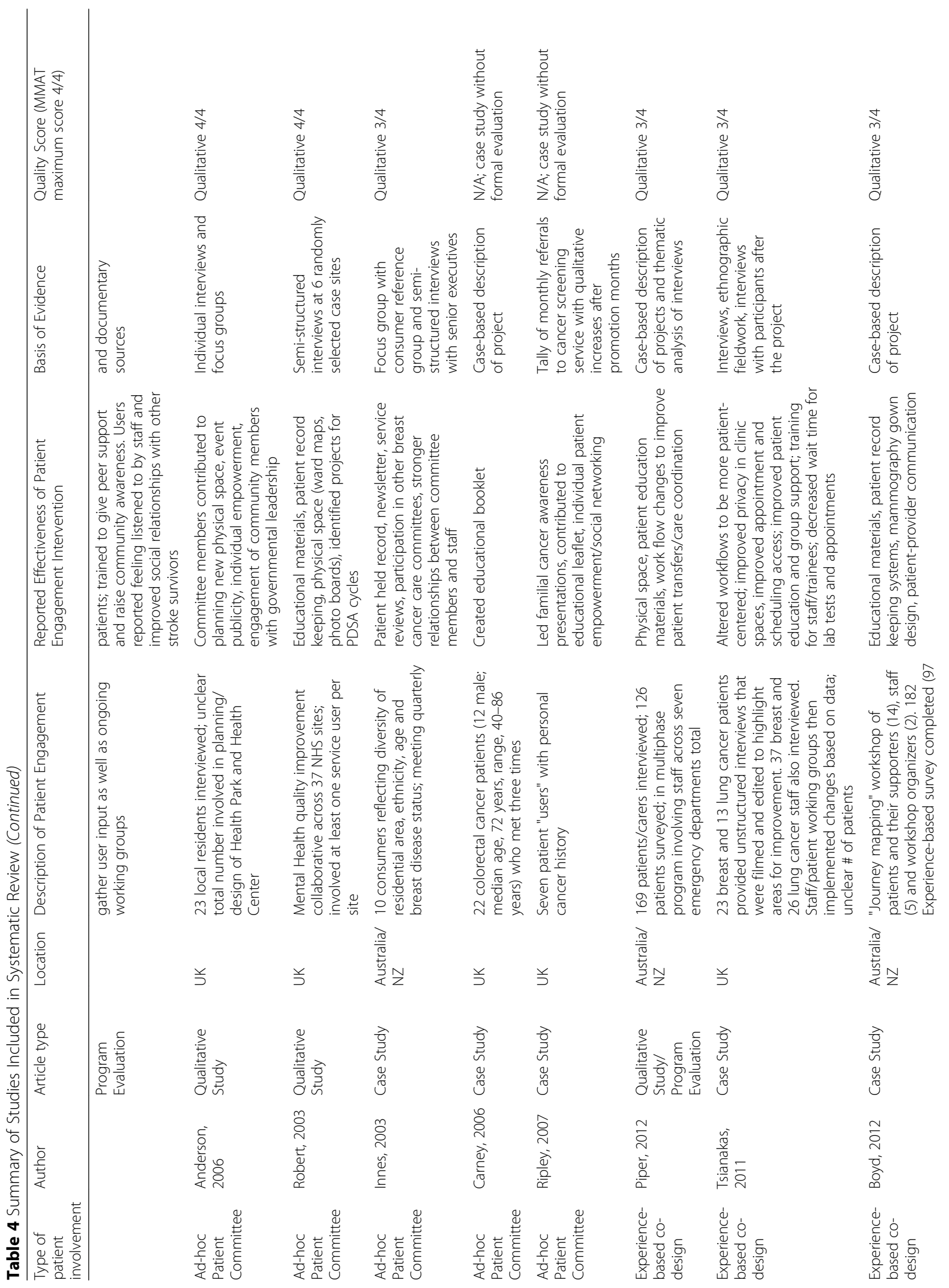


Sharma et al. BMC Health Services Research (2017) 17:693

Page 10 of 14

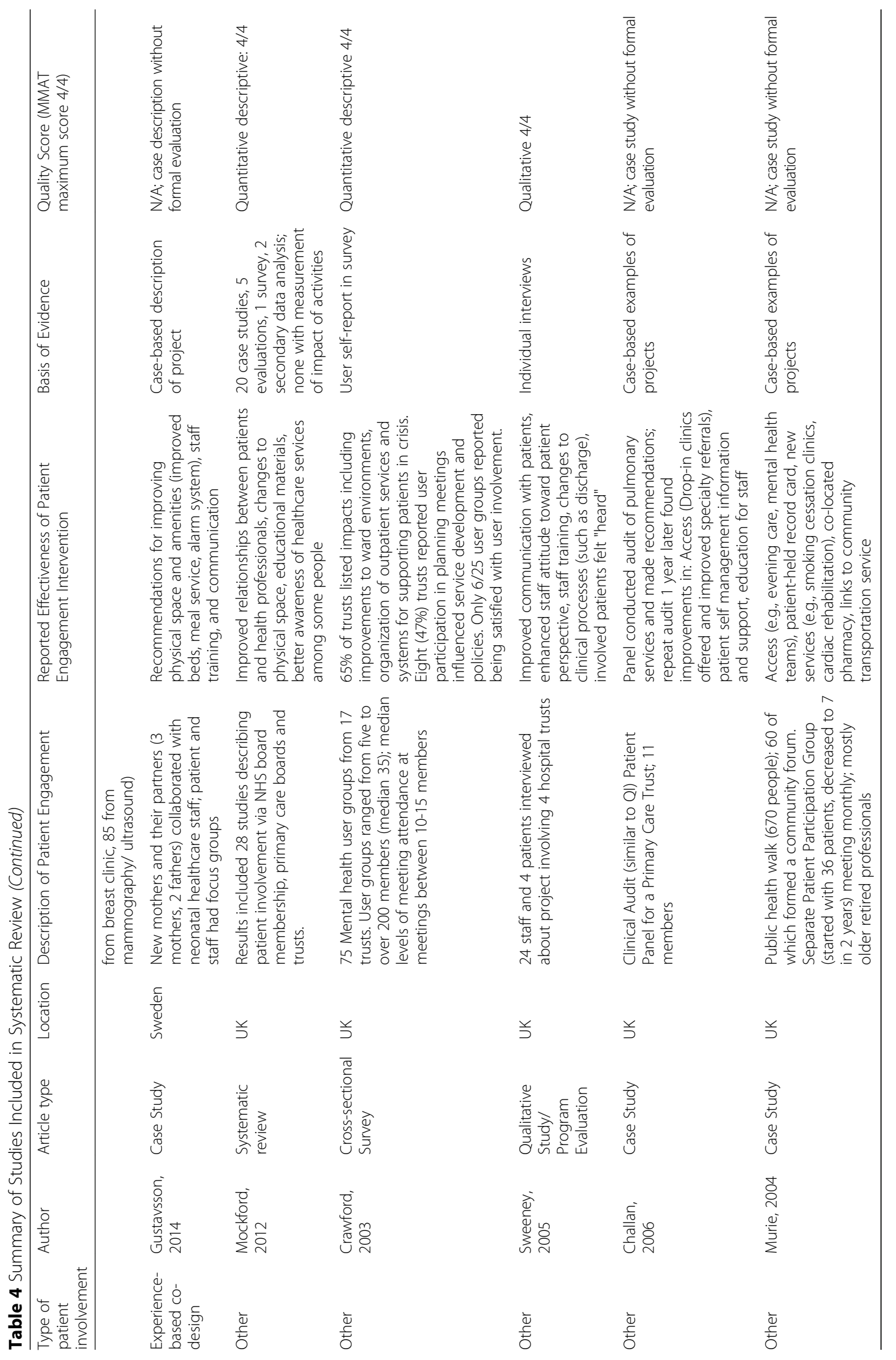


screening, asthma and hypertension control. While the studies showed statistically significant improvement in health behaviors, the control group included those who did not encounter the health promotion program at all rather than a control health promotion message without patient input. We cannot definitively attribute the improvements in health outcomes seen to the patient engagement component. We found only one clusterrandomized trial showing patient advisors helped clinics set priorities that were better aligned with the $\mathrm{PCMH}$ and chronic care model.

Despite a growing policy focus on patient-centered care, research on organizational-level patient engagement has made little progress over the 15 years that have elapsed since the publication of the 2002 Crawford systematic review. Similar to the Crawford article, we observed the most commonly found examples of advisors having an impact were on improvements to patient educational materials, clinical physical space, and changes to staff "culture" or awareness. Our review included one other systematic review of patient and public involvement in the UK, which found very similar case-based evidence within our secondary outcomes. Our review adds to these findings through our international scope and ability to capture a variety of patient advisory roles. The few experimental studies did show improvements in some clinical care metrics, clinic priorities, and staff awareness of patient engagement; however more concrete outcome measures were lacking.

Objective clinical outcomes, including quality, safety, and patient satisfaction, should be assessed in order to provide a stronger evidence base for system-level patient engagement. It is likely a challenging environment to assess these domains, given that patient advisory councils are an incredibly heterogeneous intervention. Patient advisory councils typically work on a number of projects at a given time and there are rarely joint efforts for a similar project across multiple patient advisory councils or multiple sites. Additionally, implementation of an advisory council takes significant time and resources to recruit, hold meetings, and provide follow up [53]. Implementers of advisory councils likely lack the bandwidth to incorporate formal evaluations into their work.

In order to know if patient advisory councils are actually impactful, a future research agenda for patient engagement must overcome the limitations inherent to this field. Patient advisory councils and similar patient engagement approaches are ripe for knowledge translation approach, i.e., a close, interactive relationship between researchers and health systems to accelerate evidence and improve health systems [54], Individual sites working with advisory councils should incorporate quality improvementinformed data gathering tools and consider projects as Plan-Do-Study-Act cycles with iterative analysis of improvement. For example, a hospital could assess consumer satisfaction with a waiting room space before and after an advisory council-led renovation, or assess individual patients' self-efficacy in managing diabetes before and after using a patient advisory council-devised diabetes pamphlet. Higher quality research will require concerted efforts across healthcare sites. For example, if a healthcare network implements patient advisory councils across multiple primary care sites, central leadership could encourage a shared quality goal (improvement in standardized patient satisfaction surveys, network-wide mammogram rates, etc.) which would provide an aligned outcome that could be assessed using either a pre-post analysis, stepped-wedge, or cluster-randomized design to provide a comparison group.

The study designs utilized by the few studies that did formally evaluate patient advisory councils can serve as a guidepost for future investigators interested in assessing the impact of patient engagement. The PBRN-based public health intervention partnered with community advisory council members, who were best suited for adapting and translating evidence-based health promotion materials so as to be compelling to community members [45]. If this intervention had included a study arm providing health promotion without community advisors, it would have allowed for a more rigorous analysis of how the community advisory council input improved effectiveness. In contrast, a robust control group was included in the cluster-randomized trial of an intervention working with patient advisors to set primary care priorities; by utilizing randomization, the study addressed the many potential confounders involved in clinic-level interventions. This study established a clear comparison group of staff working without patients, allowing the investigators to identify the changes in primary care priorities that could be attributed to patient involvement [22]. A comparison group was also included in a study assessing staff awareness of patient engagement before and after working with patient partners; qualitative analysis showed an expanded and improved understanding and receptivity to patient engagement in the study arm that worked with patient and family advisors [43]. By including a control arm and/or quantitative outcome measures, these studies highlight how patient advisors may enable healthcare programs to become more patient-centered.

As healthcare becomes more consumer-focused, we have much to learn from the business and industrial design world. The studies we identified exploring experience-based co-design are a promising start to incorporating the patient perspective in healthcare improvement efforts, although they did not include reports of pre- and post-evaluation of how changes 
affected the care experience, which would be helpful to understand if the co-design process should be widely disseminated.

Our study limitations include regional variations in terminology. In the US, "patient engagement" is the preferred term, while in the UK "patient and public involvement" is more common. "Co-creation" and "co-production" are growing in popularity in Australia [55]. While we may not have captured all of these variations with our search strategy, we designed a search strategy aimed to capture studies that included patients as advisors regardless of the "engagement" term used. Another limitation is possible confounding of results; some studies mentioned improvements in quality or patient satisfaction measures after largescale care redesigns across a hospital that included patient advisory councils, making it less plausible that the improvements seen could solely be attributed to the work of the patient advisors. It is also likely that positive examples of patient advisory council projects are more likely to be written up than unsuccessful ones, thus the included case studies are an example of reporting bias. The group of studies that addressed our primary outcomes of interest were from one practice-based research network in Colorado, US, thus their findings were geographically bounded in one region. Finally, we may not have captured the most recently published studies relating to patient advisory councils.

Despite these limitations, our review highlights both the strengths and challenges inherent in the field of patient engagement research. While the currently published evidence base for patient advisors is limited and primarily case-based, it does provide a description of the scope of patient advisors roles and their potential for collaboration with staff on healthcare improvement efforts, which can serve as examples for staff beginning to work with patient advisory councils. The few studies that were RCTs or quasi-experimental should serve as references for future prospective evaluations.

\section{Conclusions}

The studies included in this systematic review show how patient advisors have been involved in a wide range of projects oriented towards practice improvement. Patient advisors seem to help healthcare organizations develop programs that are more accessible or understandable which may translate to more effective primary care outcomes and priority setting. Case-based reports describe patient advisory council involvement in projects to improve clinical care and safety. Future work must seek rigorous evaluation of patient advisory council programs, in order to demonstrate value in the investments needed to implement patient-centered care.

\section{Additional files}

Additional file 1: PRISMA Checklist. PRISMA is an evidence-based set of guidelines for reporting systematic reviews. The checklist confirms that authors have followed PRISMA guidelines when completing this systematic review. (DOC 63 kb)

Additional file 2: Search Syntax for All Included Databases. The supplementary table identifies how the search strategy was adapted to return focused results from each database included in the systematic review-PubMed, Google Scholar, CINAHL, and SCOPUS (DOCX 14 kb)

Additional file 3: Mixed Methods Appraisal Tool Scores. This supplementary table includes details on the scoring rationale for our quality assessment of our final included articles using the Mixed Methods Appraisal Tool (DOCX 109 kb)

\section{Acknowledgements \\ Thank you to Dr. Evans Whitaker for informatics support during the systematic review search protocol development.}

\section{Funding}

Dr. Sharma's work was funded by NRSA Award T32HP19025. Ms. Knox's work was funded by the Kaiser Permanente National Community Benefit Fund of the East Bay Community Foundation \#20152632.

\section{Availability of data and materials}

The datasets used and/or analyzed during the current study are available from the corresponding author on reasonable request.

\section{Authors' contributions \\ All authors have reviewed and approved of the final manuscript. AES conceived of the idea for the paper, created the study search protocol, reviewed all abstracts and included papers, and wrote the majority of the manuscript. MK reviewed abstracts and full text, reviewed reference lists for articles not captured in original search, drafted portions of the Methods section, edited the manuscript, and drafted the summary table of included articles. VLM translated the search protocol for use in SCOPUS and CINAHL. He reviewed half of the abstracts and all of the included papers and drafted portions of the Methods section. JNO served as a mentor to the study investigators and also participated in the review of abstracts, resolution of conflicts on inclusion-exclusion of ab- stracts, data extraction, and manuscript review.}

Ethics approval and consent to participate

As a systematic review this study did not involve animal or human subjects and is therefore not applicable for ethics approval.

\section{Consent for publication}

Not applicable.

\section{Competing interests}

The authors have no competing interests to disclose.

\section{Publisher's Note}

Springer Nature remains neutral with regard to jurisdictional claims in published maps and institutional affiliations.

\section{Author details}

${ }^{1}$ Center for Excellence in Primary Care, Department of Family \& Community Medicine, UCSF, 995 Potrero Ave, Ward 83, San Francisco, CA 94110, USA. ${ }^{2}$ Contra Costa Regional Medical Center, Family Medicine Residency Program, 2500 Alhambra Avenue, Martinez, CA 94553, USA. ${ }^{3}$ University of California, San Francisco, 2120 University Avenue, Berkeley, CA 94704, USA.

Received: 9 May 2017 Accepted: 22 September 2017

Published online: 23 October 2017

References

1. Health Policy Brief. Patient Engagement, Health Affairs. 2013. http://www.healthaffairs.org/healthpolicybriefs/brief.php?brief_id=86. Accessed 28 Sept 2017. 
2. Carman KL, Dardess P, Maurer M, Sofaer S, Adams K, Bechtel C, et al. Patient and family engagement: a framework for understanding the elements and developing interventions and policies. Health aff (Project Hope). 2013;32(2): 223-31. doi: https://doi.org/10.1377/hlthaff.2012.1133. PubMed PMID: 23381514

3. Sharma $A E$, Grumbach K. Engaging patients in primary care practice transformation: theory, evidence and practice. Family Practice. 2017:34(3):262-7.

4. Willard-Grace RSA, Parker C, Potter MB. Engaging Patients as Partners in Practice Improvement: A Survey of Community Health Centers. J Clin Outcomes Manag. 2016;23(7):311-9.

5. American Academy of Family Physicians. Joint principles of the PatientCentered Medical Home. Del Med J. 2008;80(1):21-2. PubMed PMID: 18284087

6. National Committee for Quality Assurance. "Archive: NCQA PCMH 2011 and 2014." Last updated 16 Nov 2016. http://www.ncqa.org/programs/ recognition/practices/patient-centered-medical-home-pcmh/pcmh-archive.

7. Hastings D. Value-based payment, accountable care, and the ACO final rule: are we making progress. Health Affairs Blog [blog on the Internet]. 2011

8. Ripley M, Sullivan D, Evans J. The role of patient users in cancer genetics services in primary care. Familial Cancer. 2007;6(2):241-8.

9. Innes $L$, Bowen $L$, Bruce N, Scott $L$, and Williams P. An evaluation of consumer participation in hospital breast services. International Journal of Consumer Studies. 2003; 27(1):72-9.

10. Middleton $P$, Stanton $P$, Renouf N. Consumer consultants in mental health services: Addressing the challenges. J Ment Health. 2004;13(5):507-18.

11. Berwick DM. What 'patient-centered' should mean: confessions of an extremist. Health Aff (Millwood). 2009;28(4):w555-w565. Epub 2009/05/21. doi: https://doi.org/10.1377/hlthaff.28.4.w555. PubMed PMID: 19454528

12. Crawford MJ, Rutter D, Manley C, Weaver T, Bhui K, Fulop N, et al. Systematic review of involving patients in the planning and development of health care. BMJ. 2002, 325;(7375):1263. PubMed PMID: 12458240; PubMed Central PMCID: PMCPMC136920

13. Mockford C, Staniszewska S, Griffiths F, Herron-Marx S. The impact of patient and public involvement on UK NHS health care: a systematic review. Int J Qual Health Care. 2012;24(1):28-38. Epub 2011/11/24. doi: https://doi.org/10. 1093/intahc/mzr066. PubMed PMID: 22109631

14. Evidence Partners Incorporated. DistillerSR. Ottowa; 2015.

15. Agency for Healthcare Quality and Research. National Quality Measures Clearinghouse Rockville, MD2016 [cited 2016 November 21st] Available from: https://www.qualitymeasures.ahrq.gov/.

16. Higgins J, Altman D, Sterne J. Table 8.5. a The Cochrane Collaboration's tool for assessing risk of bias. Chapter 8: Assessing risk of bias in included studies. Cochrane handbook for systematic reviews of interventions version.510.

17. Pluye $P$, Gagnon M-P, Griffiths F, Johnson-Lafleur J. A scoring system for appraising mixed methods research, and concomitantly appraising qualitative, quantitative and mixed methods primary studies in Mixed Studies Reviews. Int J Nurs Stud. 2009;46(4):529-46.

18. Goudy E, Jackson M, Otter RD, Bater M. The journey to include patient and family voices. In Healthcare management forum. Los Angeles, CA: SAGE Publications. 2015;28(2):61-4.

19. Case study. Including patients in hospital management decisions: patients become partners in the care process. Joint Commission Perspectives on Patient Safety. 2006;6(1):9-11 2p. PubMed PMID: 106399176. Language: English. Entry Date: 20060224. Revision Date: 20150818. Publication Type: Journal Article.

20. Kamuzora P, Maluka S, Ndawi B, Byskov J, Hurtig AK. Promoting community participation in priority setting in district health systems: experiences from Mbarali district, Tanzania. Glob Health Action. 2013:6:22669. Epub 2013/11/ 28. doi: https://doi.org/10.3402/gha.v6i0.22669. PubMed PMID: 24280341; PubMed Central PMCID: PMCPmc3841300

21. McCoy DC, Hall JA, Ridge M. A systematic review of the literature for evidence on health facility committees in low- and middle-income countries. Health Policy Plan. 2012;27(6):449-66. Epub 2011/12/14. doi: https://doi.org/10.1093/heapol/czr077. PubMed PMID: 22155589

22. Boivin A, Lehoux P, Lacombe R, Burgers J, Grol R. Involving patients in setting priorities for healthcare improvement: a cluster randomized trial. Implement Sci. 2014;9:24. doi: https://doi.org/10.1186/1748-5908-9-24. PubMed PMID: 24555508; PubMed Central PMCID: PMCPMC3936906

23. Norman N, Bennett C, Cowart S, Felzien M, Flores M, Flores $R$, et al. Boot camp translation: a method for building a community of solution. J Am Board Fam Med. 2013;26(3):254-63.

24. Zittleman L, Emsermann C, Dickinson M, Norman N, Winkelman K, Linn G, et al. Increasing colon cancer testing in rural Colorado: evaluation of the exposure to a community-based awareness campaign. BMC Public Health.
2009;9:288. Epub 2009/08/12. doi: https://doi.org/10.1186/1471-2458-9-288. PubMed PMID: 19664277; PubMed Central PMCID: PMCPmc2731102

25. Bender BG, Dickinson P, Rankin A, Wamboldt FS, Zittleman L, Westfall JM. The Colorado Asthma Toolkit Program: A Practice Coaching Intervention from the High Plains Research Network. J Am Board Fam Med. 2011;24(3): 240-8. https://doi.org/10.3122/jabfm.2011.03.100171.

26. DeAlleaume L, Parnes B, Zittleman L, Sutter C, Chavez R, Bernstein J, et al. Success in the Achieving CARdiovascular Excellence in Colorado (A CARE) Home Blood Pressure Monitoring Program: A Report from the Shared Networks of Colorado Ambulatory Practices and Partners (SNOCAP). J Am Board Fam Med. 2015;28(5):548-55. https://doi.org/10.3122/jabfm.2015.05.150024.

27. Tsianakas V, Robert G, Maben J, Richardson A, Dale C, Wiseman T. Implementing patient-centred cancer care: using experience-based codesign to improve patient experience in breast and lung cancer services. Support Care Cancer. 2012;20(11):2639-47.

28. White K. Engaging patients to improve the healthcare experience. Healthc Financ Manage. 2012:66(7):84-8. PubMed PMID: 22788042

29. Ponte PR, Conlin G, Conway JB, Grant S, Medeiros C, Nies J, et al. Making Patient-centered Care Come Alive: Achieving Full Integration of the Patient's Perspective. J Nurs Adm. 2003;33(2):82-90. PubMed PMID: 00005110200302000-00004

30. Richardson A, Sitzia J, Cotterell P. 'Working the system'. Achieving change through partnership working: an evaluation of cancer partnership groups. Health Expect. 2005;8(3):210-20.

31. Challans E. How can users be involved in service improvement in health and social care, and why is this important? J Integr Pathw. 2006;10(2):49-58.

32. Murie J, Douglas-Scott G. Developing an evidence base for patient and public involvement. Clinical Governance: An Int J. 2004;9(3):147-54.

33. Meyers $\mathrm{S}$. Take heed. How patient and family advisors can improve quality. Trustee:J Hosp Gov Boards. 2008;61(4) 14-6, 21-2, 1

34. McTavish M, Phillips R. Transforming the patient experience: Bringing to life a patient-and family-centred interprofessional collaborative practice model of care at Kingston General Hospital. Patient Exp J. 2014;1(1):50-5.

35. Greenwood L. Complain and simple. Health Service Journal. 2003;113(5857): 24-5 2p. PubMed PMID: 106849383. Language: English. Entry Date: 20030711. Revision Date: 20150711. Publication Type: Journal Article.

36. Perreault M, Renaud J, Bourassa F, Beauchesne L, Mpiana A, Bernier S, et al. Implementation of a panel of service users for the evaluation of mental health outpatient services. Evaluation \& the Health Professions. 2010;33(4): 480-96 17p. doi: https://doi.org/10.1177/0163278710383561. PubMed PMID: 104966743. Language: English. Entry Date: 20110415. Revision Date: 20150711. Publication Type: Journal Article.

37. Robert G, Hardacre J, Locock L, Bate P, Glasby J. Redesigning mental health services: lessons on user involvement from the Mental Health Collaborative. Health Expect. 2003;6(1):60-71.

38. Kendell C, Urquhart R, Petrella J, MacDonald S, McCallum M. Evaluation of an advisory committee as a model for patient engagement. Patient Exp J. 2014;1(2):62-70

39. Bowen L, Richmond D. Southern Health Breast Services Consumer Participation Project Evaluation Report. 2004. http://citeseerx.ist.psu.edu/ viewdoc/download?doi=10.1.1.508.9809\&rep=rep1\&type=pdf.

40. Rich C, Goncalves A, Guardiani M, O'Donnell E, Strzelecki J. Teen Advisory Committee: lessons learned by adolescents, facilitators, and hospital staff. Pediatr Nurs. 2014;40(6):289-96. Epub 2015/05/02. PubMed PMID: 25929124

41. Anderson $E$, Shepherd M, Salisbury C. 'Taking off the suit': engaging the community in primary health care decision-making. Health Expect. 2006; 9(1):70-80. Epub 2006/01/27. doi: https://doi.org/10.1111/j.1369-7625.2006. 00364.x. PubMed PMID: 16436163; PubMed Central PMCID: PMCPmc5060321

42. Fudge N, Wolfe CD, McKevitt C. Assessing the promise of user involvement in health service development: ethnographic study. BMJ. 2008;336(7639): 313-7. Epub 2008/01/31. doi: 10.1136/bmj.39456.552257.BE. PubMed PMID: 18230646; PubMed Central PMCID: PMCPmc2234509

43. Forbat L, Cayless S, Knighting K, Cornwell J, Kearney N. Engaging patients in health care: an empirical study of the role of engagement on attitudes and action. Patient Educ Couns. 2009:74(1):84-90.

44. Sweeney G, O'Hagan B, Squire S, Powell C. The Patients Accelerating Change Project: does it make any difference? Clinical Governance: An Int J. 2005;10(1):72-83.

45. Norman N, Bennett C, Cowart S, Felzien M, Flores M, Flores $R$, et al. Boot camp translation: a method for building a community of solution. J Am 
Board Fam Med. 2013;26(3):254-63. Epub 2013/05/10. doi: https://doi.org/ 10.3122/jabfm.2013.03.120253. PubMed PMID: 23657693; PubMed Central PMCID: PMCPmc3828080

46. Loud $\mathrm{F}$, Jain $\mathrm{N}$, Thomas $\mathrm{N}$. How to develop a patient and carer advisory group in a quality improvement study. J Ren Care. 2013;39(Suppl 2):2-9. Epub 2013/08/16. doi: https://doi.org/10.1111/j.1755-6686.2013.12032.x. PubMed PMID: 23941698

47. Carney L, Jones L, Braddon F, Pullyblank A, Dixon A. A colorectal cancer patient focus group develops an information package. The Annals of The Royal College of Surgeons of England. 2006;88(5):447-9.

48. Robert G, Hardacre J, Locock L, Bate P, Glasby J. Redesigning mental health services: lessons on user involvement from the Mental Health Collaborative. Health Expect. 2003;6(1):60-71. Epub 2003/02/27. PubMed PMID: 12603629; PubMed Central PMCID: PMCPmc5060162

49. Piper D, ledema R, Gray J, Verma R, Holmes L, Manning N. Utilizing experience-based co-design to improve the experience of patients accessing emergency departments in New South Wales public hospitals: an evaluation study. Health Serv Manag Res. 2012;25(4):162-72. Epub 2013/04/05. doi: https://doi.org/10.1177/0951484812474247. PubMed PMID: 23554443

50. Boyd H, McKernon S, Mullin B, Old A. Improving healthcare through the use of co-design. New Zealand Med J (Online). 2012;125(1357)

51. Gustavsson SM. Improvements in neonatal care; using experience-based codesign. Int J Health Care Qual Assur. 2014;27(5):427-38. Epub 2014/08/05. doi: https://doi.org/10.1108/ijhcqa-02-2013-0016. PubMed PMID: 25087340

52. Crawford M, Aldridge T, Bhui K, Rutter D, Manley C, Weaver T, et al. User involvement in the planning and delivery of mental health services: a cross-sectional survey of service users and providers. Acta Psychiatr Scand. 2003;107(6):410-4.

53. Sharma A, Angel L, Bui Q. Patient Advisory Councils: Giving Patients a Seat at the Table. Fam Pract Manag. 2014;22(4):22-7.

54. CIHR. Knowledge Translation Strategy (2004-2009). Ottowa, ON: Canadian Institutes of Health Research; 2004. Available from: http://www.cihr-irsc.gc. $\mathrm{ca} / \mathrm{e} / 26574 . \mathrm{html}$

55. Janamian T, Crossland L, Wells L. On the road to value co-creation in health care: the role of consumers in defining the destination, planning the journey and sharing the drive. Med J Aust. 2016;204(7 Suppl):S12-S4.

\section{Submit your next manuscript to BioMed Central and we will help you at every step:}

- We accept pre-submission inquiries

- Our selector tool helps you to find the most relevant journal

- We provide round the clock customer support

- Convenient online submission

- Thorough peer review

- Inclusion in PubMed and all major indexing services

- Maximum visibility for your research

Submit your manuscript at www.biomedcentral.com/submit
Biomed Central 